\title{
El oráculo de Apolo en Dídima: un caso de consulta en la época helenística (Milet I 3.33)
}

\author{
The oracle of Apollo at Didyma: an oracle consultation \\ in the Hellenistic period (Milet I 3.33)
}

\author{
Federica PeZzoli*
}

\begin{abstract}
RESUMEN
El análisis de Milet I 3.33, decreto aprobado por la asamblea de Mileto en 234/233 a.C., en el que se confiere la ciudadanía a numerosos individuos, identificables como mercenarios cretenses reclutados por la ciudad, permite demostrar el papel político del oráculo de Apolo en Dídima en la época helenística.

En efecto el oráculo, estrechamente relacionado con Mileto, no sólo es un instrumento usado por la ciudad jonia para establecer relaciones con los soberanos helenísticos, especialmente con los Seléucidas, sino que es también a veces un instrumento implicado en el proceso de toma de decisiones que marca la política regional de Mileto y, al mismo tiempo, una autoridad capaz de encauzar las decisiones de la polis, ahí donde la concordia ciudadana esté en riesgo.
\end{abstract}

PALABRAS CLAVE:

Mileto, Apolo, Dídima, oráculo, helenismo, mercenarios, ciudadanía.

\begin{abstract}
The analysis of Milet I 3.33, decree of the Milesian assembly granting citizenship to Cretan immigrants, probably mercenaries, shows the political role of the oracle of Apollo at Didyma during the Hellenistic period. The oracle, closely linked to Miletos, is in fact useful in establishing and maintaining relations between the Ionian city and the Hellenistic dynasties, especially the Seleucids, but reflects at the same time the Milesian need to extend her influence in the neighbouring area, sometimes dealing with potentially complex civic matters.
\end{abstract}

\section{KEYWORDS:}

Miletos, Apollo, Didyma, oracle, Hellenism, mercenaries, citizenship.

* Dipartimento di Filologia Linguistica e Tradizione Classica «A. Rostagni», Università degli Studi di Torino (Via Sant'Ottavio 20, 10124 Torino. Italia). E-mail: federica.pezzoli @ unito.it.

Artículo basado en la comunicación leída el 27 de Mayo de 2009, en la VIII edición del Encuentro de Jóvenes Investigadores de Historia Antigua de la Universidad Complutense de Madrid. 


\section{MILETO Y LAS CIUDADES GRIEGAS DE ASIA MENOR EN ÉPOCA HELENISTICA}

En la época helenística, las ciudades de Asia Menor se mueven sobre un doble frente diplomático: por un lado, deben entablar relaciones con las grandes dinastías que controlan o luchan por el control de la región; por otro lado, en cambio, a causa de la inestabilidad política local, deben intentar establecer uniones con las póleis cercanas, pero también crear zonas de influencia sobre las que poder ejercer su autoridad y control ${ }^{1}$. Dicha duplicidad de frentes se refleja de algún modo en la actividad del santuario del oráculo de Dídima, estrechamente relacionado con Mileto, también desde el punto de vista geográfico —obsérvese la vía sacra que une el templo de Apolo Delfinio de Mileto con el templo de Apolo en Dídima-: ése no sólo tiene un importante papel en la definición de las relaciones entre la ciudad y los soberanos helenísticos, especialmente los Seléucidas ${ }^{2}$, sino que a veces también está implicado en el proceso de toma de decisiones que marca la política regional de la ciudad.

A partir de finales del siglo III a.C. y al menos hasta los primeros veinte años del siglo II a.C., Mileto, liberada del control de las potencias extranjeras, consigue conquistar una posición de hegemonía en la zona circundante, que es evidente tanto en los diferentes tratados estipulados con otras póleis más o menos cercanas $^{3}$ como en la progresiva absorción de zonas limítrofes, controladas también a través del envío de guarniciones y la creación de fuertes ${ }^{4}$. Precisamente en relación con la exigencia milesia de controlar una zona en el confín con Magnesia del Meandro y Priene, pero también de garantizarse la disponibilidad de un contingente de soldados expertos para un caso de necesidad, se inserta el documento epigráfico sobre el que tratará este artículo.

\section{EL TEXTO DEL PRIMER DECRETO SOBRE LOS MERCENARIOS CRETENSES}

El epígrafe indicado como Milet I 3.33 es un decreto aprobado por la asamblea de Mileto bajo la propuesta de un colegio de synedroi, en el que se confiere la ciudadanía a numerosos individuos, identificables como mercenarios cretenses reclutados en los años anteriores por la ciudad (frag. [f] I.10). La medida pertenece al 234/233 a.C. en base a la indicación del cargo epónimo ${ }^{5}$, y abarca un gran número de personas, como se puede deducir por la lista incompleta de los nuevos ciudadanos, Milet I 3.34, en la que aparecen registrados al menos 40 nombres de va-

1 Cf. Fernoux (2004: 115-66).

2 Paus. 1.16.3 y 8.46.3; Didyma II, 479 y II, 480. Cf. Günther (1971: 39-43); Voigtländer (1975: 2954); Parke (1985: 44-68); Fontenrose (1988: 15-20); Morgan (1989: 36-38); Parker (2000: 101-102).

3 Milet I 3.141, 143, 146, 144: tratados de isopoliteia con Cio (229/228 a.C.), Seleucia-Tralleis (218/217 a.C.), Milasa (215/214 a.C.) y Antioquía del Meandro, respectivamente. Cf. Mastrocinque (1979: 174); Herrmann (2001: 109-116).

4 Milet I 3.149, sympoliteia con Pidasa (188/187 a.C.); cf. Gauthier (2001: 117-27). Sympoliteia con Miunte, que hay que situar entre los años 289/288 y 212/211 a.C., sobre la cual ver Mazzucchi (2008: 387-407).

5 Cf. Wörrle (1988: 421-76). 
rones adultos, algunos acompañados por sus mujeres e hijos ${ }^{6}$. En base a la dimensión del muro del santuario de Apolo Delfinio en el que el decreto y la lista estaban inscritos, el primer editor, Albert Rehm, aventuraba que el número de individuos involucrados era muy superior al conservado.

El decreto, fragmentario, no permite conocer las circunstancias precisas por las que la ciudad decide realizar esta politografía de masas y de efecto inmediato; las líneas aún ahora legibles indican, sin embargo, que los beneficiarios de la medida estaban divididos en dos grupos: el primero, formado por hombres que provenían de las comunidades cretenses de Dreros y Milas o Milatos (frag. [f] I.7), el segundo, por hombres que llegaban a Mileto bajo la guía de tres hegemones, de los cuales el único nombre conservado es el de Filanor, y que probablemente también ellos eran cretenses (frag. [g] II.7-8). Los dos contingentes de soldados pidieron a la comunidad la ciudadanía, y los milesios les concedieron no sólo esto, sino también las tierras —situadas en la región de Hybandis, en la zona de Miunte- en las que podían asentarse los nuevos ciudadanos, solos o con sus familias (frags. [d] II.12-14 y [e] I.15).

Como se indica en el texto, el voto de la asamblea se alcanzó tras dos consultas al oráculo de Apolo en Dídima, registradas en el decreto y conservadas parcialmente. En la primera, relativa al grupo cretense de Dreros y Milas, la comunidad milesia preguntó al dios si la concesión de la ciudadanía, de la tierra y de la participación en todas las demás cosas en las que toman parte los ciudadanos de Mileto era la mejor decisión ${ }^{7}$; el dios contestó que sí, expresando su propia autorización en dísticos elegíacos ${ }^{8}$. En la segunda, la pregunta hecha por la ciudad es la misma, pero los beneficiarios son los soldados que llegaron con Filanor y los otros dos hegemo$n e s^{9}$; la respuesta del dios, claramente positiva, sin embargo, no se conserva - de hecho, sólo se leen algunas palabras del oráculo, también en verso-.

\section{LAS RAZONES DE LA CONSULTA DEL ORÁCULO}

¿Por qué razón la comunidad milesia recurre al consejo del oráculo de Dídima sobre una cuestión, como la concesión de la ciudadanía, que normalmente se gestiona a nivel humano ${ }^{10}$ ? La respuesta hay que buscarla en las repercusiones so-

6 Cf. Petropoulou (1985: 183-99); Chaniotis (2002: 110ss.); Loman (2004: 49-50).

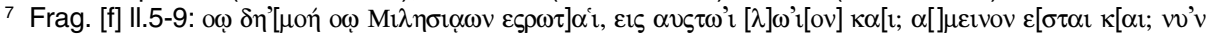

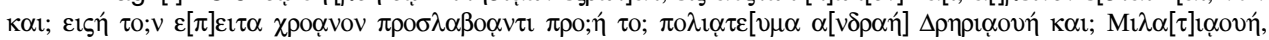

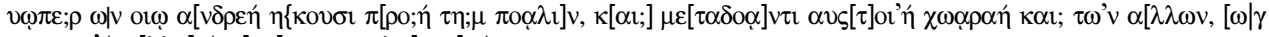

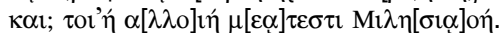

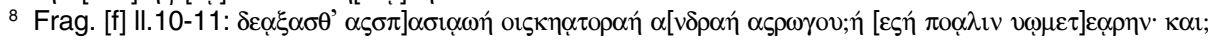
$\gamma \alpha ; \rho \lambda \omega^{\prime}$ 'ov $\kappa \alpha$; $\alpha[\mu \varepsilon$ ivov, «acoged de buena gana en vuestra ciudad como habitantes a los hombres que vienen para ayudaros; es mejor y preferible». El resto del oráculo, muy fragmentario, es incomprensible. Cf. Merkelbach y Stauber (1998: 80).

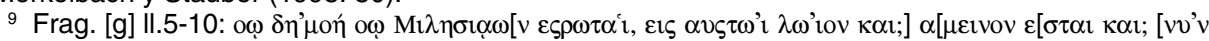

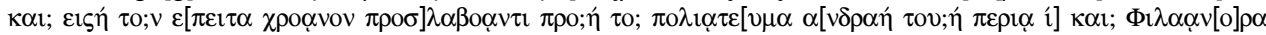

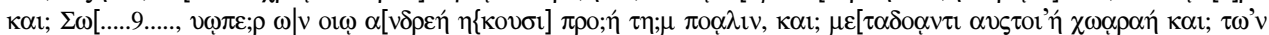

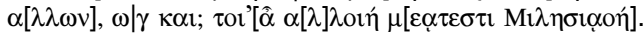

10 Cf. Morgan (1989: 31) y Parker (2000: 90). 
ciales, demográficas y políticas de la introducción de un gran número de mercenarios en la ciudad ${ }^{11}$. El mismo texto epigráfico, junto con la lista de los neopolitai, proporciona algunas indicaciones al respecto.

Ante todo, se puede afirmar que las póleis helenísticas recurren a politografías de masas - que a veces se configuran como anapleroseis, es decir, auténticas integraciones de la ciudadanía - bastante raramente ${ }^{12}$, y a menudo por razones tanto militares como demográficas: en ausencia de ciudadanos varones adultos capaces de servir como soldados, la comunidad ciudadana, por propia iniciativa o por voluntad de un soberano -es el caso de Larisa en el año 217 a.C.-_, concede la ciudadanía a individuos ya presentes en el territorio - periecos, metecos, extranjeros, mercenarios, etc. - para que participen en la defensa de la pólis. Dicha integración implica normalmente la inscripción inmediata de los beneficiarios en las divisiones cívicas existentes - tras un análisis preliminar (dokimasía) de los candidatos-y la concesión de lotes donde asentarse ${ }^{13}$. Dado que las repercusiones sociales de estos actos son tales que modifican los equilibrios internos del cuerpo cívico, es comprensible que se recurra a ellos sólo en casos de efectiva urgencia.

En el caso que estamos estudiando, Mileto, por razones históricas no del todo claras - se ha aventurado que las circunstancias que han hecho necesaria la integración de los cretenses haya sido la presencia en la zona de Antíoco lerax y de los Gálatas ${ }^{14}$, o la guerra fronteriza con Magnesia del Meandro por la posesión de Miunte ${ }^{15}$ - , se halla en la situación de conceder la ciudadanía a los mercenarios: se trata de una medida ventajosa desde el punto de vista militar y económico ${ }^{16}$, pero también capaz de generar una situación potencialmente crítica y en la que se considera, por lo tanto, necesario solicitar el beneplácito de la divinidad. Los beneficiarios, que probablemente fueron reclutados anteriormente por la ciudad -no hay que excluir la posibilidad de que el grupo procedente de Dreros y Milas haya sido enrolado por Licas de Ermofanto, uno de los synedroi que proponen el decreto y el destinatario de una estatua con inscripción en el Bouleuterion, Milet I 2.12-, de hecho se inscribirán en tres tribus (frag. [d] I.5) —en el caso de Amizonte en Caria, por ejemplo, se eligen las tribus más pequeñas (I. Amyzon 26) - y deberán jurar que defenderán a su nueva patria con celo y rapidez, continuando con el trabajo por el que los habían contratado (frag. [f] II.1-5). Algunos de estos nuevos ciudadanos, como indica la lista reproducida en Milet I 3.34, sin embargo, no se mueven solos sino con su familia, compuesta a menudo por mujer e hijos: concretamente, de los 44 nombres conservados, 14 hombres están registrados solos, 14 forman parte de una familia de dos personas, y 12 de una familia que tiene de 1 a 3 hijos $^{17}$.

11 Cf. Arist. Pol. 1303a 25-b 3 sobre las consecuencias políticas de la presencia dentro de una misma comunidad de grupos étnicos diferentes.

12 Cf. Lonis (1992: 245-70), que indica 8 casos de anaplerosis para la época helenística, pero también Launey (1987: II 650ss.).

13 Cf. Savalli (1985: 387-431).

14 Launey (1987: II 660-64).

15 Brulé (1978: 165, n.2); Herrmann (1997: 161).

16 Cf. Mazzucchi (2008: 396), que aventura que la ciudadanía es la recompensa por no haberles pagado el sueldo.

17 Cf. Petropoulou (1985: 183-88). 
Esto significa que el impacto demográfico en la comunidad milesia $-\mathrm{y}$ miusia especialmente- será claramente más consistente de lo que se supone teniendo en cuenta sólo a los varones adultos.

Además, por las líneas del decreto milesio, tal y como ha sido integrado por Rehm (frag. [c] II.6-13), se sabe que las relaciones del contingente extranjero con la población local fueron en el pasado más bien complejas: se establece, de hecho, una especie de amnistía en virtud de la cual los contenciosos de los mercenarios con los ciudadanos y viceversa ya no se podrán llevar ante el tribunal y someter a juicio. Dicha disposición muestra claramente las difíciles relaciones y la desconfianza entre los dos grupos, marcadas también por el hecho de que muchos de los soldados llevan consigo su núcleo familiar — como apunta Chaniotis ${ }^{18}$, fuera de Egipto los mercenarios normalmente no contraen matrimonio con las mujeres lugareñas, entre otros motivos para garantizar la ciudadanía legítima de sus hijos, sino que viajan con sus mujeres y concubinas o, como mucho, se casan con hijas o familiares de otros mercenarios, con cuyas patrias existen tratados de isopoliteía.

Otro factor de roce con los ciudadanos más ancianos y con la población del lugar tiene que ver con la distribución de las tierras a los recién llegados -Milet I 3.35 registraba la diaresis-: éstos últimos, de hecho, obtienen lotes de tierra en la región periférica de Hibandis, que pertenecía en un principio a la comunidad de Miunte (frag. [d] I.12) $)^{19}$, y deben cultivarlas durante al menos veinte años, con la prohibición de venderlas ${ }^{20}$. Los miusios, que tienen casas dentro del fuerte situado en esta zona -tal parece ser el significado que hay que atribuir al término $\chi \omega p$ o $\alpha_{0}{ }^{21}$ - deberán además acoger a nuevos ciudadanos y darles también alojamiento/casas en las que asentarse y suministrarles enseres y bienes muebles. Precisamente el asentamiento de los ex mercenarios cretenses, ahora ciudadanos obligados a defender la ciudad y sus confines, en un área limítrofe a la chora de Magnesia del Meandro, en la que eran frecuentes las batallas y correrías, deja clara la doble función de la politografía. Por un lado, los nuevos ciudadanos serán una especie de guarnición que controle y defienda no sólo la zona sino también toda la ciudad ${ }^{22}$, sustituyendo en esta labor a los habitantes del lugar, que quizás ya no son tan numerosos para poder ejercer eficazmente esta función. Por otra parte, irán rentabilizando los terrenos de una región cuyas condiciones ambientales estaban empeorando progresivamente. De hecho, la pequeña ciudad de Miunte, en cuyo territorio se halla Hibandis - la región que abarcaba seguramente el curso del río Hibandos y la isla de Hibanda, recordada por Plinio el Viejo 23 _, fue absorbida poco a poco por Mileto durante el siglo III a.C., hasta convertirse en uno de sus demos: todavía independiente en 289/288 a.C., cuando se la recuerda como a uno de los 13 miembros del koinón jónico, estipuló antes del año 212/211 a.C. un tratado de

18 Chaniotis (2002: 99-113).

19 El río Hibandos, afluente del Meandro, será el límite entre los territorios de Mileto y Magnesia del Meandro en el tratado de paz (Milet I 3.148, II.30-32) estipulado entre las dos póleis a finales del año 180 a.C.

20 Savalli (1985: 394-95 y 424-25).

21 Savalli (1985: 424 y n. 239) y Mazzucchi (2008: 399-400).

22 Pimouguet (1995: 97-99).

23 Robert (1969: 1437-46). 
sympoliteía con la potente vecina, después del cual gran parte de la población se trasladó a Mileto. Según fuentes antiguas ${ }^{24}$, el acercamiento y la posterior fusión con Mileto dependió sobre todo de factores ambientales: el Meandro, depositando sus sedimentos, cerró a lo largo de los años el golfo Látmico y transformó la región en un territorio pantanoso, difícil de recorrer e infectado por insectos; esto provocó con el tiempo que los miusios no pudiesen quedarse allí y que tuviesen, por lo tanto, que trasladarse a Mileto ${ }^{25}$. Volviendo a nuestro texto, parece ser que la situación no estaba tan deteriorada cuando Mileto decidió entregar a los nuevos ciudadanos cretenses los lotes de terreno en Hybandis (frag. [e] II.5-6).

Por último, cabe destacar que el decreto demuestra claramente la creación por parte de la pólis, por exigencias tanto militares como demográficas, de un auténtico barrio étnico, situado en una zona periférica, cuyos habitantes, de origen cretense, están obligados a permanecer en él al menos veinte años con sus familias, sin poder vender los lotes que las autoridades milesias les han asignado ${ }^{26}$.

Las observaciones hechas hasta ahora aclaran las dificultades que implicaba acoger a estos nuevos ciudadanos —resueltas desde el punto de vista legislativo-, pero también las ventajas que la ciudad obtenía de la politografía: soldados expertos, probablemente menos costosos que un contingente de mercenarios, destinados a una zona militarmente activa y encargados al mismo tiempo de garantizar la rentabilidad agrícola ${ }^{27}$.

La utilidad de dicha medida se confirma con el hecho de que unos años más tarde, en 229/228 a.C., Mileto concede de nuevo la ciudadanía a un nutrido contingente de soldados cretenses, muy probablemente también mercenarios, que se trasladan a Jonia con sus familias - se ha aventurado que la ciudad los había enrolado para hacer frente a la expedición de Antígono Dosón-: se trata de Milet I 3.37, decreto registrado por la comunidad dentro del mismo dossier epigráfico al que pertenecen Milet I 3.33, 34 y 35. En el probouleuma de dicho decreto, Milet I 3.36, se indica una consulta preventiva al oráculo de Dídima -(frag. [a] II.35-40), texto enteramente reconstruido-, ordenada por la ciudad, y la celebración de sacrificios en honor a Apolo de Dídima, Zeus Boulaios y Hestia Boulaia para que la concesión de la ciudadanía contribuyese a la salvación y a la concordia de la comunidad milesia. Esta vez, el texto del propio decreto, Milet I 3.37, recuerda, para justificar la medida que el pueblo ha votado, el parentesco y la familiaridad entre cretenses y milesios con motivo del $\operatorname{dios}^{28}$ y las relaciones de alianza estipuladas en el pasado y mantenidas en el presente (frag. [a] II.1-11); establece, por lo tanto, la resolución de los contenciosos jurídicos entre viejos y nuevos ciudadanos (frag. [c] II.46-50); por último organiza la distribución de los neopolitai en las tribus y la prohibición, durante veinte años, de ocupar los cargos militares de la phrurarchia y

24 Paus. 7.2.10-11; Strab. 14.1.10; Vitr. 6.1. Cf. también Herrmann (1965: 93-96).

25 Asheri (1971: 85).

26 Asheri (1971: 90-91).

27 Couvenhes (2004 : 103-104); ver Baker (2000) y Ducrey (2000) sobre el coste de los mercenarios.

28 Lücke (2000) y Musti (2001: 46-63) sobre la syngeneia y la oikeiotes. 
de la phulake ${ }^{29}$, además de la exención durante cinco años del pago de las liturgias (frag. [d] II.63-67). En este caso, sin embargo, no se especifica el lugar donde los nuevos ciudadanos obtendrán los lotes para cultivar y las casas en las que vivir no hay que excluir la idea de que hayan llegado para apoyar a sus connacionales, nombrados ciudadanos algunos años antes, en la región de Hybandis-. Además, esta segunda ola migratoria fue mucho más consistente que la primera, dado que la lista incompleta de los nuevos politai (Milet I 3.38) suma más de 200 varones adultos, casi todos acompañados por sus familias; se inscribió a los beneficiarios en la ciudadanía gradualmente, tal y como lo demuestra la división por fechas en la lista de los nombres ${ }^{30}$.

Las dos politografías estudiadas, que según Rehm demuestran que llegaron a Mileto a lo largo de cinco años unos 1.000 nuevos ciudadanos con sus familias determinando una auténtica repoblación, también militar, del territorio limítrofe con Magnesia-, no son los únicos casos en los que la ciudad se dirige al dios de Dídima para dirimir cuestiones de política local y sobre todo de integración de la propia ciudadanía. Para ello se puede citar el tratado de paz con el que se concluye, entre los años 185 y 180 a.C., la guerra fronteriza entre Mileto y Heraclea del Latmos (Milet I 3.150): las dos ciudades, antes enemigas, no sólo deciden dirimir por vía diplomática las cuestiones todavía sin resolver, sino que, a petición de los embajadores de Heraclea ${ }^{31}$, estipulan un acuerdo de isopoliteia, en virtud del cual cada uno de los ciudadanos de los dos centros contrayentes gozará de una ciudadanía potencial en el otro centro. Antes de proceder a su ratificación, las autoridades milesias sienten la necesidad de pedir la opinión de Apolo de Dídima, que expresa su opinión favorable (II.16-17). Gauthier ${ }^{32}$ considera que ya se ha llevado a cabo el mismo iter algunos años antes, en 188/187 a.C., para la aprobación de la sympoliteía con Pidasa, que llevó a la absorción política de esta pequeña comunidad por parte de Mileto: precisamente dado que también la sympoliteía implica la concesión de la ciudadanía milesia a los pidaseos, aunque es probable que éstos no hayan dejado su comunidad de origen, es bastante posible que se haya pedido opinión al oráculo de Dídima y que éste haya dado su aprobación -la razón por la cual los pidaseos solicitaron y obtuvieron la sympoliteía sería probablemente de carácter militar-defensivo-.

\section{ALGUNAS CONCLUSIONES}

Los casos analizados permiten sacar algunas conclusiones.

El oráculo de Apolo en Dídima es, al contrario que el oráculo de Apolo en Delfos, un oráculo 'ciudadano', cuyos acontecimientos en época histórica están estrechamente relacionados con los de la ciudad de Mileto. Esto explica por qué,

29 Savalli (1985: 425).

30 Brulé (1990: 236-52).

31 Gauthier (2001: 124)

32 Gauthier (2001: 120-22). 
frente a la crisis que sufre Delfos en el periodo helenístico, el santuario del oráculo de Dídima vive un auténtico renacimiento, contemporáneo al de la ciudad patrona, ya sea como instrumento usado por Mileto para establecer relaciones con los soberanos helenísticos, ya sea como autoridad capaz de encauzar las decisiones de la pólis ahí donde la concordia ciudadana esté en riesgo ${ }^{33}$. Por lo tanto, no es casual que quienes consulten el oráculo sean a menudo, además de cada uno de los ciudadanos, los magistrados de la ciudad.

Esto confirma el papel político de los oráculos tal y como ha aventurado la tradición crítica más reciente: las póleis consultan a los oráculos sobre problemas que no pueden resolverse sólo por medios humanos, pero también sobre temas cuyas repercusiones pueden recaer en la comunidad a diferentes niveles. Por consiguiente, las prescripciones e indicaciones de los oráculos se tienen en gran consideración en sede deliberativo-legislativa, es decir, cuando la ciudad, recibido el oráculo, aprueba una decisión sobre la cuestión ${ }^{34}$.

A veces la estrecha relación entre Dídima y Mileto, visible también físicamente en la vía sacra que va del templo de Apolo Delfinios al de Apolo de Dídima, se demuestra con el hecho de que los oráculos dados por el dios reflejan las relaciones existentes entre Mileto y las demás ciudades jónicas cercanas ${ }^{35}$ — hay que pensar en los ex-mercenarios cretenses asentados, con el beneplácito de Apolo, en el confín con el territorio de Magnesia del Meandro-.

Para concluir, una curiosidad: como ya se ha indicado anteriormente, los oráculos registrados en el primer decreto de concesión de la ciudadanía a los mercenarios cretenses están escritos en dísticos elegíacos. Dado que los oráculos fragmentarios de época arcaica son en prosa ${ }^{36}$, es probable que con la reanudación de la actividad en torno al año 331 a.C. el oráculo didimeo haya adaptado su propio funcionamiento al del oráculo délfico: de ahí el uso de una sacerdotisa como voz y trámite de Apolo y la presencia de oráculos escritos en verso, destinados a preservarse en el tiempo ${ }^{37}$.

\section{BIBLIOGRAFÍA}

Milet I 3 (1914) = Das Delphinion in Milet, II. Die Inschriften, von A. Rehm, Berlin. Didyma II (1958) = Didyma, II. Die Inschriften, von A. Rehm, Berlin.

ASHERI, D. (1971), «Supplementi coloniari e condizione giuridica della terra nel mondo greCO», RSA 1, 77-91.

BAKER, P. (2000), “Coûts des garnisons et fortifications dans les cités à l'époque hellénistique», en Andreau, J.; Briant, P. y Descat, R. eds., Guerre et Économie dans les Sociétés Antiques, Saint-Bertrand-des-Comminges, 177-196.

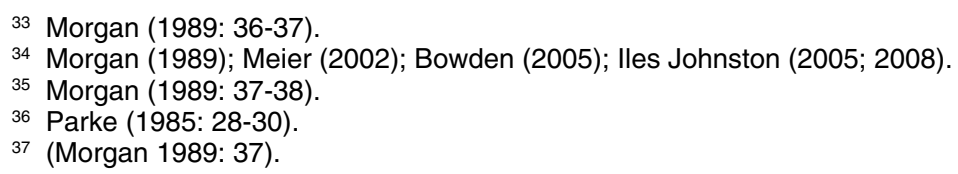


- (2003), “La guerre à l'époque hellénistique», en Prost, F. ed., L'Orient Méditerranéen de la Mort d'Alexandre aux Campagnes de Pompée, Rennes, 381-401.

BOWDEN, H. (2005), Classical Athens and the Delphic Oracle, Cambridge.

BRULÉ, P. (1978), La Piraterie Cretoise Hellénistique, Paris.

- (1990), «Enquête démographique sur la famille grecque antique. Études de listes de politographie d'Asie Mineure», REA 92.2, 233-258.

CHANIOTIS, A. (2002), «Foreign soldiers - native girls? Constructing and crossing boundaries in Hellenistic cities with foreign garrisons", en Chaniotis, A. y Ducrey, P. eds., Army and Power in the Ancient World, Stuttgart, 99-113.

- (2004), «Mobility of persons during the Hellenistic wars: state control and personal relations", en Moatti, C. ed., La Mobilité de Personnes en Méditerranée de l'Antiquité à l'Époque Moderne, Roma, 481-500.

COUVENHES, J. C. (2004), "Les cités grecques d'Asie Mineure et le mercenariat», en Couvenhes, J. C. y Fernoux, H. L. eds., Les Cités Grecques et la Guerre en Asie Mineure à l'Époque Hellénistique, Tours, 77-106.

DUCREY, P. (2000), Les Aspects Économiques de l'Usage de Mercenaires dans la Guerre en Grèce Ancienne, en Andreau, J.; Briant, P. y Descat, R. eds., Guerre et Économie dans les Sociétés Antiques, Saint-Bertrand-des-Comminges, 197-210.

FERNOUX, H. L. (2004), «Les cités s'entraident dans la guerre: historique, cadres institutionnels et modalités pratiques des conventions d'assistance dans l'Asie mineure hellénistique», en Couvenhes, J.C. y Fernoux, H.L. eds., 115-166.

FONTENROSE, J. (1988), Didyma. Apollo's Oracle, Cult and Companion, Berkeley.

GAUTHIER, P. (2001), «Les Pidaséens entrent en sympolitie avec les Milésiens: la procédure et les modalités institutionelles», en Bresson, A. y Descat, R. eds., Les Cites d'Asie Mineure Occidentale au I/e Siècle a.C., Bordeaux, 117-127.

GÜNTHER, W. (1971), Das Orakel von Didyma in hellenistischer Zeit, Tübingen.

HERRMANN, P. (1965), «Neue Urkunden zur Geschichte von Milet im 2. Jahrhundert v. Chr.», MDAI(I) 15, 71-117.

- (1997), Milet VI.1. Inschriften von Milet. B. Nachträge und Übersetzungen zu den Inschriften n. 1-406, Berlin.

- (2001), «Milet au II siècle a.C.», en Bresson, A. y Descat, R. eds., Les Cites d'Asie Mineure Occidentale au Ile Siècle a.C., Bordeaux, 109-116.

ILES JOHNSTON, S. (2005), "Introduction: divining divination», en lles Johnston, S. y Struck, P. eds., Mantike. Studies in Ancient Divination, Boston.

- (2008), Ancient Greek Divination, Oxford.

LAUNEY, M. (1987), Recherches sur les Armées Hellénistiques, 2 vols., Paris.

LOMAN, P. (2004), «No woman no war: women's participation in ancient Greek warfare», G\&R 51, 34-54.

LONIS, R. (1992), «L'anaplérosis ou la reconstitution du corp civique avec des étrangeres à l'époque hellénistique», en Lonis, R. ed., L'Étranger dans le Monde Grec II, Nancy, 245-270.

LÜCKE, S. (2000), Syngeneia. Epigraphisch-historische Studien zu einem Phänomen der antiken griechischen Diplomatie, Frankfurt am Main.

MAZZUCCHI, R. (2008), «Mileto e la sympoliteia con Miunte», en Virgilio, B. ed., Studi Ellenistici. $X X$, Roma, 387-407.

MEIER, J. K. (2002), «Omens, prophecies and oracles in ancient decision-making», en Ancient History Matters. Studies Presented to Jens Erik Skydsgaard on his Seventieth Birthday, Roma, 173-183

MERKELBACH, R. y STAUBER, J. (1998), Steinepigramme aus dem griechischen Osten, Leipzig.

MORGAN, C. (1989), «Divination and society at Delphi and Didyma», Hermathena 147, $17-$ 42.

MUSTI, D. (2001), «La syngheneia e la oikeiotes: sinonimi o nuances?», en Angeli BertinelIi, M. G. y Piccirilli, L. eds., Serta Antiqua et Mediaevalia IV, Roma, 43-63. 
PARKE, H. W. (1985), The Oracles of Apollo in Asia Minor, London.

PARKER, R. (2000), "Greek states and Greek oracles», en Buxton, R. ed., Oxford Readings in Greek religion, Oxford, 76-105.

PETROPOULOU, A. (1985), Beiträge zur Wirtschafts- und Gesellschaftsgeschichte Kretas in hellenistischer Zeit, Frankfurt am Main.

PIMOUGUET, I. (1995), «Défense et territoire: l'exemple milésien», DHA 21.1, 89-109.

ROBERT, L. (1969), «Philologie et géographie II. Sur Pline l'Ancien livre II», en Robert, L., Opera Minora selecta, vol. III, Amsterdam, 1437-1446.

SAVALLI, I. (1985), «I neocittadini nelle città ellenistiche. Nota sulla concessione della cittadinanza e l'acquisizione della politeia», Historia 34, 387-431.

VOIGTLÄNDER, W. (1975), Der jüngste Apollontempel von Didyma, Tübingen.

WÖRRLE, M. (1988), «Inschriften von Herakleia am Latmos I: Antiochos, Zeuxis und Herakleia», Chiron 18, 421-476. 